\title{
Complexity of Reasoning over Temporal Data Models
}

\author{
A. Artale ${ }^{1}$, R. Kontchakov ${ }^{2}$, V. Ryzhikov ${ }^{1}$, M. Zakharyaschev ${ }^{2}$ \\ ${ }^{1}$ Faculty of Computer Science \\ Free University of Bozen-Bolzano \\ I-39100 Bolzano, Italy \\ lastnamedinf.unibz.it \\ ${ }^{2}$ Department of Computer Science \\ Birkbeck College \\ London WC1E 7HX, UK \\ \{roman, michael\}@dcs.bbk.ac.uk
}

\begin{abstract}
We investigate the computational complexity of reasoning over temporal extensions of conceptual data models. The temporal conceptual models we analyse include the standard UML/EER ISA between entities and relationships, disjointness and covering, cardinality constraints and their refinements, multiplicity and key constraints; in the temporal dimension, we have timestamping, evolution and transition constraints, as well as lifespan cardinalities. We give a nearly comprehensive picture of the impact of these constraints on the complexity of reasoning, which can range from NLOGSPACE to undecidability.
\end{abstract}

\section{Introduction}

Temporal conceptual data models $[25,24,14,15,19,4,23,12]$ extend standard conceptual schemas with means to visually represent temporal constraints imposed on temporal database instances. According to the glossary [18], such constraints can be divided into three categories, to illustrate which we use the temporal data model in Fig. 1.

Timestamping constraints are used to discriminate between those elements of the model that change over time - they are called temporary — and others that are timeinvariant, or snapshot. Timestamping is realised by marking entities, relationships and attributes by $\mathrm{T}$ (for temporary) or $\mathrm{S}$ (for snapshot), which is then translated into a timestamping mechanism of the database. In Fig. 1, Employee and Department are snapshot entities, Name and PaySlipNumber are snapshot attributes and Member a snapshot relationship. On the other hand, Manager is a temporary entity, Salary a temporary attribute and Works $\mathrm{n}$ a temporary relationship. If no timestamping constraint is imposed on an element, it is left unmarked (e.g., Manages).

Evolution and transition constraints control permissible changes of database states $[13,23,7]$. For entities, we talk about object migration from one entity to another [17]. Transition constraints presuppose that migration happens in a fixed amount of time. For example, the dashed arrow marked by TEX in Fig. 1 means that each Project expires in exactly one year. On the other hand, evolution constraints are qualitative in the sense that they do not restrict the length of migration. In Fig. 1, an AreaManager will eventually become a TopManager (the dashed arrow marked by DEV), each Manager was once an Employee (DEX ${ }^{-}$), and a Manager cannot be demoted (PEX).

Evolution-related knowledge can also be conveyed through generation relationships [16,23]. For instance, the generation relationship Propose between Manager 


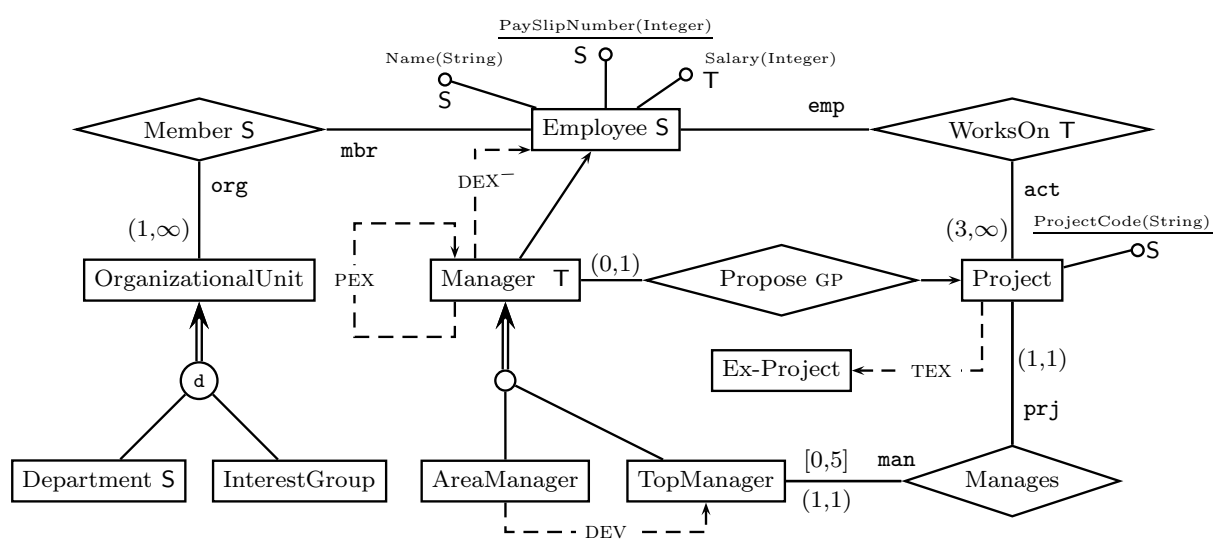

Fig. 1. An $\mathcal{E} \mathcal{R}_{V T}$ temporal conceptual data model.

and Project (marked by GP, with an arrow pointing to Project) in Fig. 1 means that managers may create new projects.

Lifespan cardinality constraints $[14,23,24]$ are temporal counterparts of standard cardinality constraints. While the latter restrict the number of times an object can participate in a relationship and are evaluated at each moment of time, lifespan cardinality constraints are evaluated over the entire existence of the object. According to Fig. 1, for example, every member of TopManager can manage up to five different projects in its entire existence, but exactly one project at each moment of time.

The temporal conceptual model $\mathcal{E R}_{V T}$ we consider in this paper is a generalisation of the formalisms introduced in [4,7]. Apart from the temporal constraints discussed above, it includes the standard UML/EER constructs: ISA, disjointness (circled d in Fig. 1) and covering (double arrow) constraints between entities and relationships, cardinality constraints and their refinements, as well as multiplicity constraints for attributes and key constraints for entities. The language of $\mathcal{E} \mathcal{R}_{V T}$ and its model-theoretic semantics are defined in Section 2. This formalisation of temporal conceptual models also provides us with a rigorous definition of various quality properties of temporal conceptual schemas. For instance, a minimal quality requirement for a schema is its consistency in the sense that its constraints are not contradictory-or, semantically, have at least one (nonempty) model. We may also need guarantees that some entities and relationships in the schema are not necessarily empty (at some or all moments of time) or that one entity (relationship) is not subsumed by another one. To automatically check such quality properties, it is essential to provide an effective reasoning support during the construction phase of a temporal conceptual model.

The main aim of this paper is to investigate the impact of various types of temporal and atemporal constraints on the computational complexity of checking quality properties of $\mathcal{E R}_{V T}$ temporal conceptual models. First, we distinguish between the full (atemporal) EER language $E R_{\text {full }}$, its fragment $E R_{\text {bool }}$ where ISA can only be used for en- 
tities (but not relationships), and the fragment $E R_{\text {ref }}$ thereof where covering constraints are not available. Reasoning in these (non-temporal) languages is known to be, respectively, EXPTIME-, NP- and NLOGSPACE-complete [10,2]. We then combine each of these EER data models with the temporal constraints discussed above and give a nearly comprehensive classification of their computational behaviour. Of the obtained complexity results summarised in Fig. 2 we emphasise here the following:

- It is known [1] that timestamping and evolution constrains together cause undecidability of reasoning over $E R_{\text {full }}$ (EXPTIME-completeness, if timestamping is restricted to entities [5]). We show in Section 3, however, that reasoning becomes only NP-complete if the underlying EER language is restricted to $E R_{\text {bool }}$ or $E R_{\text {ref }}$.

- Timestamping and transition constraints over $E R_{b o o l}$ result in NP-complete reasoning; the addition of evolution constraints increases the complexity to PSPACE.

- Evolution constraints over $E R_{\text {ref }}$ give NP-complete reasoning; transition constraints result in PTIME, while timestamping over the same $E R_{\text {ref }}$ gives only NLOGSPACE.

- Reasoning with both lifespan cardinalities and timestamping is known to be EXPTIME-complete for $E R_{\text {full }}$ [8]. We show that for $E R_{\text {bool }}$ restricted to binary relationships the problem becomes NP-complete.

- Reasoning with lifespan cardinalities and transition (or evolution) constraints is undecidable over both $E R_{\text {full }}$ and $E R_{\text {bool }}$.

We prove these results by exploiting the tight correspondence between conceptual modelling formalisms and description logics (DLs) [10,2], the family of knowledge representation formalisms tailored towards effective reasoning about structured class-based information [9]. DLs form a basis of the Web Ontology Language OWL, which is now in the process of being standardised by the W3C in its second edition OWL 2 . We show in Section 3 how temporal extensions of DLs (see [21] for a recent survey) can be used to encode the different temporal constraints and thus to provide complexity results for reasoning over temporal data models.

\section{The Temporal Conceptual Model $\mathcal{E} \mathcal{R}_{V T}$}

To give a formal foundation to temporal conceptual models, we describe here how to associate a textual syntax and a model-theoretic semantics with an EER/UML modelling language. In particular, we consider the temporal EER model $\mathcal{E} \mathcal{R}_{V T}$ generalising the formalisms of $[4,7]$ (here VT stands for valid time). $\mathcal{E} \mathcal{R}_{V T}$ supports timestamping for entities, attributes and relationships, as well as evolution constraints and lifespan cardinalities. It is upward compatible (by preserving the non-temporal semantics of conventional (legacy) conceptual schemas) and sanpshot reducible [20,23] (at each moment of time, all atemporal constraints are verified by the database described by a given temporal schema). $\mathcal{E} \mathcal{R}_{V T}$ is equipped with both textual and graphical syntax along with a model-theoretic semantics as a temporal extension of the EER semantics [11]. We illustrate the formal definition of $\mathcal{E} \mathcal{R}_{V T}$ using the schema in Fig. 1.

Throughout, by an $X$-labelled $n$-tuple over $Y$ we mean any sequence of the form $\left\langle x_{1}: y_{1}, \ldots, x_{n}: y_{n}\right\rangle$, where $x_{i} \in X$ with $x_{i} \neq x_{j}$ if $i \neq j$, and $y_{i} \in Y$.

\footnotetext{
${ }^{1}$ http://www.w3.org/2007/owL/
} 
A signature is a quintuple $\mathcal{L}=(\mathcal{E}, \mathcal{R}, \mathcal{U}, \mathcal{A}, \mathcal{D})$ consisting of disjoint finite sets $\mathcal{E}$ of entity symbols, $\mathcal{R}$ of relationship symbols, $\mathcal{U}$ of role symbols, $\mathcal{A}$ of attribute symbols and $\mathcal{D}$ of domain symbols. Each relationship $R \in \mathcal{R}$ is assumed to be equipped with some $k \geq 1$, the arity of $R$, and a $k$-tuple $\operatorname{REL}(R)=\left\langle U_{1}: E_{1}, \ldots, U_{k}: E_{k}\right\rangle$, where $E_{i} \in \mathcal{E}$ and $U_{i} \in \mathcal{U}$ with $U_{i} \neq U_{j}$ for $i \neq j$. For example, the binary relationship WorksOn in Fig. 1 has two roles: emp ranging over Employee, and act ranging over Project. Each entity $E \in \mathcal{E}$ comes equipped with a tuple $\operatorname{ATT}(E)=$ $\left\langle A_{1}: D_{1}, \ldots, A_{h}: D_{h}\right\rangle$, for some $h \geq 0$, where $A_{i} \in \mathcal{A}, D_{i} \in \mathcal{D}$ and $A_{i} \neq A_{j}$ for $i \neq j$. For example, the entity Employee in Fig. 1 has three attributes: Name is of type String while both PaySlipNumber and Salary are of type Integer. Domain symbols $D \in \mathcal{D}$ are assumed to be associated with pairwise disjoint countably infinite sets $B_{D}$ called basic domains. In Fig. 1, basic domains are the set of integer numbers (for Integer) and the set of strings (for String).

A temporal interpretation of signature $\mathcal{L}$ is a structure of the form

$$
\mathcal{I}=\left((\mathbb{Z},<), \Delta^{\mathcal{I}}, \Lambda^{\mathcal{I}},\left\{\cdot{ }^{\mathcal{I}(t)} \mid t \in \mathbb{Z}\right\}\right),
$$

where $(\mathbb{Z},<)$ is the intended flow of time, $\Delta^{\mathcal{I}} \neq \emptyset$ is the interpretation domain, $\Lambda^{\mathcal{I}}=$ $\bigcup_{D \in \mathcal{D}} \Lambda_{D}^{\mathcal{I}}$ with $\Lambda_{D}^{\mathcal{I}} \subseteq B_{D}$ and $\Delta^{\mathcal{I}} \cap \Lambda^{\mathcal{I}}=\emptyset$ is the active domain and ${ }^{\mathcal{I}(t)}$, for $t \in \mathbb{Z}$, is the interpretation function which assigns a set $E^{\mathcal{I}(t)} \subseteq \Delta^{\mathcal{I}}$ to each $E \in \mathcal{E}$, a set $R^{\mathcal{I}(t)}$ of $\mathcal{U}$-labelled tuples over $\Delta^{\mathcal{I}}$ to each relationship $R \in \mathcal{R}$, and a binary relation $A^{\mathcal{I}(t)} \subseteq \Delta^{\mathcal{I}} \times \Lambda^{\mathcal{I}}$ to each attribute $A \in \mathcal{A}$ in such a way that the following conditions are satisfied for all $t \in \mathbb{Z}$ :

- if $\operatorname{ATT}(E)=\left\langle A_{1}: D_{1}, \ldots, A_{h}: D_{h}\right\rangle, e \in E^{\mathcal{I}(t)}$ and $(e, a) \in A_{i}^{\mathcal{I}(t)}, 1 \leq i \leq h$, then $a \in \Lambda_{D_{i}}^{\mathcal{I}}$;

- if $\operatorname{REL}(R)=\left\langle U_{1}: E_{1}, \ldots, U_{k}: E_{k}\right\rangle$ and $r \in R^{\mathcal{I}(t)}$ then $r=\left\langle U_{1}: e_{1}, \ldots, U_{k}: e_{k}\right\rangle$ for $e_{i} \in E_{i}^{\mathcal{I}(t)}, 1 \leq i \leq k$.

An $\mathcal{E} \mathcal{R}_{V T}$ conceptual schema of signature $\mathcal{L}$ is a finite set of constraints imposed on temporal interpretations $\mathcal{I}$ of signature $\mathcal{L}$. We group these constraints as follows.

Generalisation / specialisation hierarchies (with disjointness and covering) on both entities and relationships:

- $E_{1}$ ISA $E_{2}$, for entities $E_{1}$ and $E_{2}$, is satisfied in $\mathcal{I}$ if $E_{1}^{\mathcal{I}(t)} \subseteq E_{2}^{\mathcal{I}(t)}$, for all $t \in \mathbb{Z}$. Similarly, $R_{1}$ ISA $R_{2}$, for relationships $R_{1}$ and $R_{2}$, is satisfied in $\mathcal{I}$ if $R_{1}^{\mathcal{I}(t)} \subseteq R_{2}^{\mathcal{I}(t)}$, for all $t \in \mathbb{Z}$. In Fig. 1, Manager IS A Employee.

- $E_{1}$ DISJ $E_{2}$ and $R_{1}$ DISJ $R_{2}$ are satisfied in $\mathcal{I}$ if $E_{1}^{\mathcal{I}(t)} \cap E_{2}^{\mathcal{I}(t)}=\emptyset$ and, respectively, $R_{1}^{\mathcal{I}(t)} \cap R_{2}^{\mathcal{I}(t)}=\emptyset$, for all $t \in \mathbb{Z}$. In Fig. 1, disjointness is indicated by a circled $d$; that Department and InterestGroup are disjoint sub-entities of OrganisationalUnit is represented by DepartmentISAOrganisationalUnit, InterestGroup ISA OrganisationalUnit, Department DISJ InterestGroup.

- $\left\{E_{1}, \ldots, E_{n}\right\} \operatorname{COV} E$ and $\left\{R_{1}, \ldots, R_{n}\right\} \operatorname{COV} R$ are satisfied in $\mathcal{I}$ if, respectively, $E^{\mathcal{I}(t)}=\bigcup_{i=1}^{n} E_{i}^{\mathcal{I}(t)}$ and $R^{\mathcal{I}(t)}=\bigcup_{i=1}^{n} R_{i}^{\mathcal{I}(t)}$, for all $t \in \mathbb{Z}$. The double arrow in Fig. 1 indicates that AreaManager and TopManager cover the entity Manager. 
It will be convenient for us to assume that the constraint $\left\{E_{1}, \ldots, E_{n}\right\} \operatorname{COV} E$ always comes together with the (implied) constraints $E_{i}$ ISA $E$ and that the ISA-constraints are transitive (i.e., if $E_{1}$ ISA $E_{2}$ and $E_{2}$ ISA $E_{3}$ then we have $E_{1}$ ISA $E_{3}$ as well). The same concerns ISA for relationships. These assumptions increase the size of the schema at most quadratically, which has no effect on our complexity results.

\section{Cardinality, multiplicity and key constraints:}

- Let $\operatorname{REL}(R)=\left\langle U_{1}: E_{1}, \ldots, U_{k}: E_{k}\right\rangle$. For $1 \leq i \leq k$, the cardinality constraint $\operatorname{CARD}_{R}\left(R, U_{i}, E_{i}\right)=(\alpha, \beta)$ with $\alpha \in \mathbb{N}$ and $\beta \in \mathbb{N} \cup\{\infty\}$ is satisfied in $\mathcal{I}$ if, for all $e \in E_{i}^{\mathcal{I}}$ and $t \in \mathbb{Z}$,

$$
\alpha \leq \sharp\left\{\left(e_{1}, \ldots, e_{i}, \ldots, e_{k}\right) \in R^{\mathcal{I}(t)} \mid e_{i}=e\right\} \leq \beta .
$$

TopManager in Fig. 1 Manages exactly one Project.

- Let $\operatorname{REL}(R)=\left\langle U_{1}: E_{1}, \ldots, U_{k}: E_{k}\right\rangle$ and $E_{i}^{\prime}$ ISA $E_{i}$, for some $1 \leq i \leq k$. The refinement $\operatorname{REF}\left(R, U_{i}, E_{i}^{\prime}\right)=(\alpha, \beta)$ of the cardinality constraint is satisfied in $\mathcal{I}$ if (1) holds for all $e \in\left(E_{i}^{\prime}\right)^{\mathcal{I}(t)}$ and $t \in \mathbb{Z}$.

- Let $\operatorname{ATt}(E)=\left\langle A_{1}: D_{1}, \ldots, A_{h}: D_{h}\right\rangle$. For $1 \leq i \leq h$, the multiplicity constraint $\operatorname{CARD}_{A}\left(A_{i}, E\right)=(\alpha, \beta)$ with $\alpha \in \mathbb{N}$ and $\beta \in \mathbb{N} \cup\{\infty\}$ is satisfied in $\mathcal{I}$ if we have $\alpha \leq \sharp\left\{a \in \Lambda_{D_{i}}^{\mathcal{I}} \mid(e, a) \in A_{i}^{\mathcal{I}(t)}\right\} \leq \beta$, for all $e \in E^{\mathcal{I}(t)}$ and $t \in \mathbb{Z}$. If the multiplicity is not given explicitly (as in Fig. 1), it is assumed to be $(1,1)$.

- Let $\operatorname{ATT}(E)=\left\langle A_{1}: D_{1}, \ldots, A_{h}: D_{h}\right\rangle$. The key constraint $\operatorname{KeY}(E)=A_{i}$ is satisfied in $\mathcal{I}$ if $\operatorname{CARD}_{A}\left(E, A_{i}\right)=(1,1)$ is satisfied in $\mathcal{I}$ and, for all $a$ in $\Lambda^{\mathcal{I}}$ and $t \in \mathbb{Z}$, we have $\sharp\left\{e \in \Delta^{\mathcal{I}} \mid(e, a) \in A^{\mathcal{I}(t)}\right\} \leq 1$. The underlined attribute PaySlipNumber in Fig. 1 is a key for Employee. Though PaySlipNumber in Fig. 1 is also a time-invariant attribute this is not always the case for key attributes.

If $\left(U_{i}, E_{i}\right)$ is not an element of $\operatorname{REL}(R)$ then $\operatorname{CARD}_{R}\left(R, U_{i}, E_{i}\right)$ is syntactically illegal (similarly for the other constraints). We assume that all numbers are given in binary.

Tmestamping constraints (TS). The set of entity symbols $\mathcal{E}$ can be partitioned into sets of snapshot $\left(\mathcal{E}^{\mathrm{S}}\right)$, temporary $\left(\mathcal{E}^{\top}\right)$ and implicitly temporal $\left(\mathcal{E}^{\mathbf{l}}\right)$ entities:

- for all $E \in \mathcal{E}^{\mathcal{S}}$ and $t \in \mathbb{Z}$, if $e \in E^{\mathcal{I}(t)}$ then $e \in E^{\mathcal{I}\left(t^{\prime}\right)}$ for all $t^{\prime} \in \mathbb{Z}$;

- for all $E \in \mathcal{E}^{\top}$ and $t \in \mathbb{Z}$, if $e \in E^{\mathcal{I}(t)}$ then there is $t^{\prime} \in \mathbb{Z}$ with $e \notin E^{\mathcal{I}\left(t^{\prime}\right)}$;

- implicitly temporal entities have no restrictions on their interpretation.

Similar partitions can be made on the sets of relationship and attribute symbols. In Fig. 1, Employee is a snapshot entity (marked by S), Manager a temporary entity (marked by T) and Project an implicitly temporal one (unmarked). PaySlipNumber and Name are snapshot attributes that do not change their values over time, whereas Salary changes over time, and so is a temporary attribute. Member is a snapshot relationship meaning that an employee is always member of the same organisational unit, while WorksOn is temporary meaning that employees can work on different projects at different times.

Evolution constraints (EVO) are grouped into dynamic evolution and persistent evolution constraints. There are three types of dynamic evolution constraints for entities: 
- $E_{1}$ DEV $E_{2}$ is satisfied in $\mathcal{I}$ if, for each $e \in E_{1}^{\mathcal{I}(t)}, t \in \mathbb{Z}$, there exists $t^{\prime}>t$ such that $e \in E_{2}^{\mathcal{I}\left(t^{\prime}\right)}$ and $e \notin E_{1}^{\mathcal{I}\left(t^{\prime}\right)}$.

- $E_{1}$ DEX $E_{2}$ is satisfied in $\mathcal{I}$ if, for each $e \in E_{1}^{\mathcal{I}(t)}, t \in \mathbb{Z}$, there exists $t^{\prime}>t$ with $e \in E_{2}^{\mathcal{I}\left(t^{\prime}\right)}$.

- $E_{1} \mathrm{DEX}^{-} E_{2}$ is satisfied in $\mathcal{I}$ if, for each $e \in E_{1}^{\mathcal{I}(t)}, t \in \mathbb{Z}$, there exists $t^{\prime}<t$ such that $e \in E_{2}^{\mathcal{I}\left(t^{\prime}\right)}$.

In Fig. 1, AreaManager DEV TopManager means that every AreaManager will eventually migrate to TopManager and Manager $\mathrm{DEX}^{-}$Employee means that every Manager was once an Employee.

There are also three types of persistent evolution constraints for entities:

- $E_{1}$ PEV $E_{2}$ is satisfied in $\mathcal{I}$ if, for each $e \in E_{1}^{\mathcal{I}(t)}, t \in \mathbb{Z}$, we have $e \in E_{2}^{\mathcal{I}\left(t^{\prime}\right)}$ and $e \notin E_{1}^{\mathcal{I}\left(t^{\prime}\right)}$, for all $t^{\prime}>t$.

- $E_{1} \operatorname{PEX} E_{2}$ is satisfied in $\mathcal{I}$ if, for each $e \in E_{1}^{\mathcal{I}(t)}, t \in \mathbb{Z}$, we have $e \in E_{2}^{\mathcal{I}\left(t^{\prime}\right)}$, for all $t^{\prime}>t$.

- $E_{1} \mathrm{PEX}^{-} E_{2}$ is satisfied in $\mathcal{I}$ if, for each $e \in E_{1}^{\mathcal{I}(t)}, t \in \mathbb{Z}$, we have $e \in E_{2}^{\mathcal{I}\left(t^{\prime}\right)}$ for all $t^{\prime}<t$.

In Fig. 1, Manager PEX Manager reflects the persistent status of Manager (once a manager, always a manager).

Transition constraints (TRANS) are of three types:

- $E_{1}$ TEV $E_{2}$ is satisfied in $\mathcal{I}$ if, for each $e \in E_{1}^{\mathcal{I}(t)}, t \in \mathbb{Z}$, we have $e \in E_{2}^{\mathcal{I}(t+1)}$ and $e \notin E_{1}^{\mathcal{I}(t+1)}$.

- $E_{1}$ TEX $E_{2}$ is satisfied in $\mathcal{I}$ if, for each $e \in E_{1}^{\mathcal{I}(t)}, t \in \mathbb{Z}$, we have $e \in E_{2}^{\mathcal{I}(t+1)}$.

- $E_{1} \mathrm{TEX}^{-} E_{2}$ is satisfied in $\mathcal{I}$ if, for each $e \in E_{1}^{\mathcal{I}(t)}, t \in \mathbb{Z}$, we have $e \in E_{2}^{\mathcal{I}(t-1)}$.

In Fig. 1, Project TEX Ex-Project means that every Project will expire in one year.

Lifespan cardinality constraints (LFC) and their refinements are defined as follows:

- Let $\operatorname{REL}(R)=\left\langle U_{1}: E_{1}, \ldots, U_{k}: E_{k}\right\rangle$. For $1 \leq i \leq k$, the lifespan cardinality constraint L-CARD $\left(R, U_{i}, E_{i}\right)=(\alpha, \beta)$ with $\alpha \in \mathbb{N}$ and $\beta \in \mathbb{N} \cup\{\infty\}$ is satisfied in $\mathcal{I}$ if, for all $t \in \mathbb{Z}$ and $e \in E_{i}^{\mathcal{I}(t)}$,

$$
\alpha \leq \sharp\left[\bigcup_{t^{\prime} \in \mathbb{Z}}\left\{\left(e_{1}, \ldots, e_{i}, \ldots, e_{k}\right) \in R^{\mathcal{I}\left(t^{\prime}\right)} \mid e_{i}=e\right\}\right] \leq \beta .
$$

In Fig. 1, TopManager can Manage at most five distinct Projects throughout the whole life.

- Let $\operatorname{REL}(R)=\left\langle U_{1}: E_{1}, \ldots, U_{k}: E_{k}\right\rangle$ and $E_{i}^{\prime}$ ISA $E_{i}$, for some $1 \leq i \leq k$. The refinement $\operatorname{L-REF}\left(R, U_{i}, E_{i}^{\prime}\right)=(\alpha, \beta)$ of the lifespan cardinality constraint is satisfied in $\mathcal{I}$ if (2) holds for all $t \in \mathbb{Z}$ and $e \in\left(E_{i}^{\prime}\right)^{\mathcal{I}(t)}$.

Generation relationships (GEN) are a sort of evolution constraints conveyed through relationships. Suppose $R$ is a binary relationship with $\operatorname{REL}(R)=\left\langle\mathrm{s}: E_{1}, \mathrm{t}: E_{2}\right\rangle$, where $s$ and $t$ are two fixed role symbols. 
- A production relationship constraint $\mathrm{GP}(R)=R^{\prime}$, where $R^{\prime}$ is a fresh relationship with $\operatorname{REL}\left(R^{\prime}\right)=\left\langle\mathrm{s}: E_{1}, \mathrm{t}: E_{2}^{\prime}\right\rangle$ and $E_{2}^{\prime}$ a fresh entity (i.e., $R^{\prime}$ do not $E_{2}^{\prime}$ occur in other constraints), is satisfied in $\mathcal{I}$ if, for all $t \in \mathbb{Z}$, we have $E_{2}^{\mathcal{I}(t)} \cap\left(E_{2}^{\prime}\right)^{\mathcal{I}(t)}=\emptyset$ and if $r=\left\langle e_{1}, e_{2}\right\rangle \in\left(R^{\prime}\right)^{\mathcal{I}(t)}$ then $e_{1} \in E_{1}^{\mathcal{I}(t)}$ and $e_{2} \in\left(E_{2}^{\prime}\right)^{\mathcal{I}(t)} \cap E_{2}^{\mathcal{I}(t+1)}$. In Fig. 1, the fact that Managers can create at most one new Project at a time is captured by constraining Propose to be a production relationship (marked by GP) together with the $(0,1)$ cardinality constraint.

- The transformation relationship constraint $\mathrm{GT}(R)=R^{\prime}$, where $R^{\prime}$ is a fresh relationship with $\operatorname{REL}\left(R^{\prime}\right)=\left\langle\mathrm{s}: E_{1}, \mathrm{t}: E_{2}^{\prime}\right\rangle$ and $E_{2}^{\prime}$ a fresh entity, is satisfied in $\mathcal{I}$ if, for all $t \in \mathbb{Z}$, we have $E_{2}^{\mathcal{I}(t)} \cap\left(E_{2}^{\prime}\right)^{\mathcal{I}(t)}=\emptyset$ and if $r=\left\langle e_{1}, e_{2}\right\rangle \in\left(R^{\prime}\right)^{\mathcal{I}(t)}$ then $e_{1} \in E_{1}^{\mathcal{I}(t)}, e_{2} \in\left(E_{2}^{\prime}\right)^{\mathcal{I}(t)} \cap E_{2}^{\mathcal{I}(t+1)}$ and $e_{1} \notin E_{1}^{\mathcal{I}\left(t^{\prime}\right)}$, for all $t^{\prime}>t$.

Note that the production relationship constraint $\operatorname{GP}(R)=R^{\prime}$ can be equivalently replaced with the disjointness and evolution constraints $E_{2}^{\prime}$ DISJ $E_{2}$ and $E_{2}^{\prime}$ TEX $E_{2}$. Similarly, the transformation relationship constraint $\mathrm{GT}(R)=R^{\prime}$ can be equivalently replaced with $\operatorname{REL}\left(R^{\prime}\right)=\left\langle\mathrm{s}: E_{1}^{\prime}, \mathrm{t}: E_{2}^{\prime}\right\rangle, E_{2}^{\prime} \mathrm{DISJ} E_{2}, E_{2}^{\prime} \mathrm{TEX} E_{2}, E_{1}^{\prime} \mathrm{ISA} E_{1}, E_{1}^{\prime} \mathrm{PEX} E_{1}^{\prime \prime}$ and $E_{1}^{\prime \prime}$ DISJ $E_{1}$, where $E_{1}^{\prime}$ and $E_{1}^{\prime \prime}$ are fresh entities. Therefore, in what follows we do not consider generation relationship constraints.

\subsection{Reasoning Problems}

The basic reasoning problems over temporal data models we are concerned with in this paper are entity, relationship and schema consistency, and subsumption for entities and relationships. To define these problems, suppose that $\mathcal{L}=(\mathcal{E}, \mathcal{R}, \mathcal{U}, \mathcal{A}, \mathcal{D})$ is a signature, $E_{1}, E_{2} \in \mathcal{E}, R_{1}, R_{2} \in \mathcal{R}$ and $\Sigma$ is an $\mathcal{E} \mathcal{R}_{V T}$ schema over $\mathcal{L}$. $\Sigma$ is said to be consistent if there exists a temporal interpretation $\mathcal{I}$ over $\mathcal{L}$ satisfying all the constraints from $\Sigma$ and such that $E^{\mathcal{I}(t)} \neq \emptyset$, for some $E \in \mathcal{E}$ and $t \in \mathbb{Z}$. In this case we also say that $\mathcal{I}$ is a model of $\Sigma$. The entity $E_{1}$ (relationship $R_{1}$ ) is consistent with respect to $\Sigma$ if there exists a model $\mathcal{I}$ of $\Sigma$ such that $E_{1}^{\mathcal{I}(t)} \neq \emptyset$ (respectively, $R_{1}^{\mathcal{I}(t)} \neq \emptyset$ ), for some $t \in \mathbb{Z}$. The entity $E_{1}$ (relationship $R_{1}$ ) is subsumed by the entity $E_{2}$ (relationship $R_{2}$ ) in $\Sigma$ if any model of $\Sigma$ is also a model of $E_{1}$ ISA $E_{2}$ (respectively, $R_{1}$ ISA $R_{2}$ ).

It is well known that the reasoning problems of checking schema, entity and relationship consistency, as well as entity and relationship subsumption are reducible to each other (see $[10,2]$ for more details). Note, however, that if the covering constructor is not available, to check schema consistency we have to run, in the worst case, as many entity satisfiability checks as the number of entities in the schema. In what follows, we only consider the entity consistency problem.

\subsection{Complexity of Reasoning}

We investigate the complexity of reasoning not only for full $\mathcal{E} \mathcal{R}_{V T}$ but also for various sub-languages obtained by weakening either the EER or the temporal component. We consider the three EER fragments identified in [2]:

- $E R_{\text {full }}$ contains all the ERR constraints. 


\begin{tabular}{|l|l|l|l|}
\hline \multirow{2}{*}{\multicolumn{1}{c|}{$\begin{array}{c}\text { temporal } \\
\text { features }\end{array}$}} & \multicolumn{3}{c|}{ EER component } \\
\cline { 2 - 5 } & $E R_{\text {full }}$ & $E R_{\text {bool }}$ & $E R_{\text {ref }}$ \\
\hline \hline TS & 2EXPTIME [8] & NP [Th.2] & NLOGSPACE [Th.5] \\
\hline TRANS & EXPTIME [5] & PSPACE [Th.1] & in PTIME [Th.4] \\
\hline TS, TRANS & UNDEC. [Th.6] & PSPACE [Th.1] & in PTIME [Th.4] \\
\hline EVO & EXPTIME [5] & NP [Th.2] & NP [Th.3] \\
\hline TS, EVO & UNDEC. [1] & NP [Th.2] & NP [Th.3] \\
\hline TRANS, EVO & EXPTIME [5] & PSPACE [Th.1] & NP [Th.3] \\
\hline TS, TRANS, EVO & UNDEC. [1] & PSPACE [Th.1] & NP [Th.3] \\
\hline \hline TS, LFC & 2EXPTIME [8] & NP ${ }^{\dagger}[$ Th.7] & in NP NTh.7] $^{\dagger}$ [Th. \\
\hline TRANS, LFC & UNDEC. [Th.8] & UNDEC. [Th.8] $^{\text {Und }}$ & $?$ \\
\hline EVO, LFC & UNDEC. [Th.6] & $?$ & $?$ \\
\hline
\end{tabular}

$\left(^{\dagger}\right)$ This result is proved only for binary relationships.

Fig. 2. Complexity of reasoning in fragments of $\mathcal{E} \mathcal{R}_{V T}$.

- $E R_{\text {bool }}$ has ISA only between entities; it is also required that attributes do not change their types: if $(A, D) \in \operatorname{ATT}(E)$ and $\left(A, D^{\prime}\right) \in \operatorname{ATT}\left(E^{\prime}\right)$, for $E, E^{\prime} \in \mathcal{E}$, then $D^{\prime}=D$.

- $E R_{\text {ref }}$ is the fragment of $E R_{\text {bool }}$ without covering constraints.

Reasoning in these (non-temporal) languages is, respectively, EXPTIME-, NP- and NLOGSPACE-complete [10,2].

Fig. 2 summarises the complexity results known in the literature or to be proved below. Unless otherwise indicated, the complexity bounds are tight.

In the subsequent sections, we denote the languages by explicitly indicating their EER and temporal components. For example, $E R_{\text {full }}^{\text {TS,EVO,TRANS,LFC }}$ denotes the full conceptual modelling language $\mathcal{E R}_{V T}$.

\section{Embedding Temporal $E R_{b o o l / r e f}$ in Temporal DL-Lite bool/core}

We prove the positive (i.e., decidability) results in the table above by reducing reasoning over temporal data models based on $E R_{b o o l}$ and $E R_{\text {ref }}$ without lifespan cardinality constraints to reasoning in temporal description logics based on variants of DL-Lite [3, 6] (in fact, these temporal DL-Lite logics were originally designed with the aim of capturing temporal data models).

The language of $T_{F P X} D L-L i t e_{\text {bool }}^{\mathcal{N}}$ contains concept names $A_{0}, A_{1}, \ldots$, local role names $P_{0}, P_{1}, \ldots$ and rigid role names $G_{0}, G_{1}, \ldots$ Roles $R$, basic concepts $B$ and concepts $C$ are defined as follows:

$$
\begin{array}{lll}
S & ::=P_{i} \mid G_{i}, & R::=S \mid S^{-}, \\
B & ::=\perp\left|A_{i}\right| \geq q R, & \\
C & ::=B|\neg C| C_{1} \sqcap C_{2}\left|\diamond_{F} C\right| \square_{F} C\left|\diamond_{P} C\right| \square_{P} C\left|\bigcirc_{F} C\right| \bigcirc_{P} C,
\end{array}
$$

where $q \geq 1$ is a natural number (given in binary). We use the construct $C_{1} \sqcup C_{2}$ as a standard Boolean abbreviation, and also set $\otimes C=\diamond_{P} \diamond_{F} C$ and $\circledast C=\square_{P} \square_{F} C$. 
A $T_{F P X} D L-$ Lite $_{\text {bool }}^{\mathcal{N}}$ interpretation $\mathcal{I}$ is a function

$$
\mathcal{I}(n)=\left(\Delta^{\mathcal{I}}, A_{0}^{\mathcal{I}(n)}, \ldots, P_{0}^{\mathcal{I}(n)}, \ldots, G_{0}^{\mathcal{I}(n)}, \ldots\right), \quad n \in \mathbb{Z},
$$

where $\Delta^{\mathcal{I}} \neq \emptyset, A_{i}^{\mathcal{I}(n)} \subseteq \Delta^{\mathcal{I}}$ and $P_{i}^{\mathcal{I}(n)}, G_{i}^{\mathcal{I}(n)} \subseteq \Delta^{\mathcal{I}} \times \Delta^{\mathcal{I}}$ with $G_{i}^{\mathcal{I}(n)}=G_{i}^{\mathcal{I}(m)}$, for all $m \in \mathbb{Z}$. The role and concept constructs are interpreted in $\mathcal{I}$ as follows:

$$
\begin{array}{rlrl}
\left(S^{-}\right)^{\mathcal{I}(n)} & =\left\{(y, x) \mid(x, y) \in S^{\mathcal{I}(n)}\right\}, \quad \perp^{\mathcal{I}(n)}=\emptyset, \quad(\neg C)^{\mathcal{I}(n)}=\Delta^{\mathcal{I}} \backslash C^{\mathcal{I}(n)}, \\
\left(C_{1} \sqcap C_{2}\right)^{\mathcal{I}(n)} & =C_{1}^{\mathcal{I}(n)} \cap C_{2}^{\mathcal{I}(n)}, & & (\geq q R)^{\mathcal{I}(n)}=\left\{x \mid \sharp\left\{y \mid(x, y) \in R^{\mathcal{I}(n)}\right\} \geq q\right\}, \\
\left(\diamond_{F} C\right)^{\mathcal{I}(n)} & =\bigcup_{k>n} C^{\mathcal{I}(k)}, & & \left(\diamond_{P} C\right)^{\mathcal{I}(n)}=\bigcup_{k<n} C^{\mathcal{I}(k)}, \\
\left(\square_{F} C\right)^{\mathcal{I}(n)} & =\bigcap_{k>n} C^{\mathcal{I}(k)}, & & \left(\square_{P} C\right)^{\mathcal{I}(n)}=\bigcap_{k<n} C^{\mathcal{I}(k)}, \\
\left(\bigcirc_{F} C\right)^{\mathcal{I}(n)} & =C^{\mathcal{I}(n+1)}, & & \left(\bigcirc_{P} C\right)^{\mathcal{I}(n)}=C^{\mathcal{I}(n-1)} .
\end{array}
$$

It follows that $(\otimes C)^{\mathcal{I}(n)}=\bigcup_{k \in \mathbb{Z}} C^{\mathcal{I}(k)}$ and $\circledast C=\bigcap_{k \in \mathbb{Z}} C^{\mathcal{I}(k)}$.

A $T_{F P X} D L-L i t e_{\text {bool }}^{\mathcal{N}} T B o x, \mathcal{T}$, is a finite set of concept inclusions (CIs) $C_{1} \sqsubseteq C_{2}$. (As usual, instead of two CIs $C_{1} \sqsubseteq C_{2}$ and $C_{2} \sqsubseteq C_{1}$ we write $C_{1} \equiv C_{2}$.) An interpretation $\mathcal{I}$ is a model of $\mathcal{T}$ if $C_{1}^{\mathcal{I}(n)} \subseteq C_{2}^{\mathcal{I}(n)}$ for all $n \in \mathbb{Z}$ and all CIs $C_{1} \sqsubseteq C_{2}$ in $\mathcal{T}$. A concept $C$ is satisfiable with respect to $\mathcal{T}$ if there exist a model $\mathcal{I}$ of $\mathcal{T}$ and $n \in \mathbb{Z}$ such that $C^{\mathcal{I}(n)} \neq \emptyset$. Concept satisfiability w.r.t. $T_{F P X} D L-L i t e_{b o o l}^{\mathcal{N}}$ TBoxes is PSPACEcomplete [6].

We now show that entity consistency w.r.t. $E R_{\text {bool }}^{\text {TS,TRANS,EVO }}$ schemas can be reduced to concept satisfiability w.r.t. $T_{F P X} D L-L i t e_{b o o l}^{\mathcal{N}}$ TBoxes. Note that since $E R_{b o o l}$ has no ISA between relationships, without loss of generality we may assume that different relations cannot share the same role in their RELS.

Suppose we are given an $E R_{\text {bool }}^{\text {TS,TRANSEVO }}$ schema $\Sigma$. All entity, relationship and domain symbols in $\Sigma$ will be regarded as concept names in the $T_{F P X} D L-L i t e_{\text {bool }}^{\mathcal{N}}$ TBox $\mathcal{T}_{\Sigma}$ we are about to construct. All role and attribute symbols in $\Sigma$ will be regarded as role names in $\mathcal{T}_{\Sigma}$. We define $\mathcal{T}_{\Sigma}$ as $\mathcal{T}_{\Sigma}^{0} \cup \mathcal{T}_{\Sigma}^{1}$, where $\mathcal{T}_{\Sigma}^{0}$ encodes the atemporal constructs and $\mathcal{T}_{\Sigma}^{1}$ the temporal ones. $\mathcal{T}_{\Sigma}^{0}$ contains the following CIs (cf. [2]):

(D) $D \sqsubseteq \neg C$, for $C \neq D$, where $D \in \mathcal{D}$ and $C \in \mathcal{E} \cup \mathcal{R} \cup \mathcal{D}$;

(A) for $\operatorname{ATT}(E)=\left\langle A_{1}: D_{1}, \ldots, A_{h}: D_{h}\right\rangle$ in $\Sigma$ and $1 \leq i \leq h$,

- $\exists A_{i}^{-} \sqsubseteq D_{i}$,

- $E \sqsubseteq \geq \alpha A_{i}$ and $E \sqsubseteq \leq \beta A_{i}$ if $\operatorname{CARD}_{A}\left(A_{i}, E\right)=(\alpha, \beta)$;

(R) for $\operatorname{REL}(R)=\left\langle U_{1}: E_{1}, \ldots, U_{k}: R_{k}\right\rangle$ in $\Sigma$ and $1 \leq i \leq k$,

- $R \equiv \exists U_{i}, \geq 2 U_{i} \sqsubseteq \perp$ and $\exists U_{i}^{-} \sqsubseteq E_{i}$,

- $E_{i} \sqsubseteq \geq \alpha U_{i}^{-}$and $E_{i} \sqsubseteq \leq \beta U_{i}^{-}$if $\operatorname{CARD}_{R}\left(R, U_{i}, E_{i}\right)=(\alpha, \beta)$,

- $E_{i}^{\prime} \sqsubseteq \geq \alpha U_{i}^{-}$and $E_{i}^{\prime} \sqsubseteq \leq \beta U_{i}^{-}$if $\operatorname{REF}\left(R, U_{i}, E_{i}^{\prime}\right)=(\alpha, \beta)$;

(H) $E_{1} \sqsubseteq E_{2}$, for $E_{1}$ ISA $E_{2}$ in $\Sigma$,

$E_{1} \sqsubseteq \neg E_{2}$, for $E_{1}$ DISJ $E_{2}$ in $\Sigma$,

$E \equiv \bigsqcup_{i=1}^{n} E_{i}$, for $\left\{E_{1}, \ldots, E_{n}\right\} \operatorname{Cov} E$ in $\Sigma$

(concept inclusions $E \sqsubseteq \geq \alpha S$ with $\alpha=0$ and $E \sqsubseteq \leq \beta S$ with $\beta=\infty$ are not included in the TBox). The temporal part $\mathcal{T}_{\Sigma}^{1}$ contains the following CIs: 
(TS) $E \sqsubseteq \otimes E$, for snapshot $E \in \mathcal{E}^{S}$, and $\top \sqsubseteq \otimes \neg E$, for temporary $E \in \mathcal{E}^{\top}$,

$R \sqsubseteq \otimes R$ and roles $U_{i}, 1 \leq i \leq k$, are rigid, for snapshot $R \in \mathcal{R}^{\mathrm{S}}$ with $\operatorname{REL}(R)=$ $\left\langle U_{1}: E_{1}, \ldots U_{k}: E_{k}\right\rangle$, the role $A$ is rigid, for snapshot $A \in \mathcal{A}^{\mathrm{S}}$;

(TRANS) $E_{1} \sqsubseteq \bigcirc_{F} E_{2}$ and $E_{1} \sqsubseteq \bigcirc_{F} \neg E_{1}$ for $E_{1} \operatorname{TEV} E_{2}$,

$E_{1} \sqsubseteq \bigcirc_{F} E_{2}$ for $E_{1}$ TEX $E_{2}$,

$E_{1} \sqsubseteq \mathrm{O}_{P} E_{2}$ for $E_{1} \mathrm{TEX}^{-} E_{2}$;

(EVO) $E_{1} \sqsubseteq \diamond_{F}\left(E_{2} \sqcap \neg E_{1}\right)$ for $E_{1} \operatorname{DEV} E_{2}$, and $E_{1} \sqsubseteq \square_{F} E_{2} \sqcap \square_{F} \neg E_{1}$ for $E_{1}$ PEV $E_{2}$, $E_{1} \sqsubseteq \diamond_{F} E_{2}$ for $E_{1} \operatorname{DEX} E_{2}$, and $E_{1} \sqsubseteq \square_{F} E_{2}$ for $E_{1} \operatorname{PEX} E_{2}$,

$E_{1} \sqsubseteq \diamond_{P} E_{2}$ for $E_{1} \mathrm{DEX}^{-} E_{2}$, and $E_{1} \sqsubseteq \square_{P} E_{2}$ for $E_{1} \mathrm{PEX}^{-} E_{2}$.

It should be clear that the size of $\mathcal{T}_{\Sigma}$ is polynomial in the size of $\Sigma$.

Lemma 1. An entity $E$ is consistent w.r.t. a $E R_{\text {bool }}^{\mathrm{TS}, \mathrm{TRANS}, \mathrm{EVO}}$ schema $\Sigma$ if and only if $E$ is satisfiable w.r.t. to the $T_{F P X} D L-L i e_{\text {bool }}^{\mathcal{N}}$ TBox $\mathcal{T}_{\Sigma}$.

The proof of this lemma uses the DL-Lite encoding of atemporal EER schemas [2] and the following lemma showing that we can treat temporary relationships and attributes as implicitly temporal:

Lemma 2. Let $\Sigma$ be an $E R_{\text {bool }}^{\mathrm{TS} \text { TRANS, EVO }}$ schema. If an entity $E$ is consistent w.r.t. $\Sigma$ then it is consistent w.r.t. the schema $\Sigma^{\prime}$, which coincides with $\Sigma$ except that all implicitly temporal relationships and attributes of $\Sigma$ are temporary in $\Sigma^{\prime}$.

Proof. Let $R_{1}$ be an implicitly temporal relationship in $\Sigma$. Mark $R_{1}$ as a temporary relationship and denote the resulting schema by $\Sigma_{1}$. Let $\mathcal{I}$ be a model of $\Sigma$ such that $E^{\mathcal{I}(t)} \neq \emptyset$ for some $t \in \mathbb{Z}$. Consider the interpretation $\mathcal{J}$ for $\Sigma_{1}$ obtained by taking the disjoint union of two copies of $\mathcal{I}$ and then setting

$R_{1}^{\mathcal{J}(t)}= \begin{cases}\left\{\left\langle e_{1}^{\prime \prime}, e_{2}^{\prime}, \ldots, e_{k}^{\prime}\right\rangle,\left\langle e_{1}^{\prime}, e_{2}^{\prime \prime}, \ldots, e_{k}^{\prime \prime}\right\rangle \mid\left\langle e_{1}, e_{2}, \ldots, e_{k}\right\rangle \in R_{1}^{\mathcal{I}(t)}\right\}, & \text { if } t=0 \\ \left\{\left\langle e_{1}^{\prime}, e_{2}^{\prime}, \ldots, e_{k}^{\prime}\right\rangle,\left\langle e_{1}^{\prime \prime}, e_{2}^{\prime \prime}, \ldots, e_{k}^{\prime \prime}\right\rangle \mid\left\langle e_{1}, e_{2}, \ldots, e_{k}\right\rangle \in R_{1}^{\mathcal{I}(t)}\right\}, & \text { otherwise }\end{cases}$

where $e_{i}^{\prime}$ and $e_{i}^{\prime \prime}$ are the two copies of $e_{i} \in \Delta^{\mathcal{I}}, 1 \leq i \leq k$. Clearly, $R_{1}$ is interpreted as a temporary relationship in $\mathcal{J}$ (all other symbols are interpreted in the same way as in $\mathcal{I})$. So, it remains to show that the REL, CARD $R$ and REF constraints involving $R_{1}$ are satisfied in $\mathcal{J}$.

$\operatorname{REL}\left(R_{1}\right)=\left\langle U_{1}: E_{1}, \ldots, U_{k}: E_{k}\right\rangle$ is satisfied in $\mathcal{J}$ for every $t \neq 0$ since $\mathcal{J}$ agrees with the disjoint union of two copies of $\mathcal{I}$. Consider $\left\langle e_{1}^{\prime \prime}, e_{2}^{\prime}, \ldots, e_{k}^{\prime}\right\rangle \in R_{1}^{\mathcal{J}(0)}$. By the construction, $\left\langle e_{1}, e_{2}, \ldots, e_{k}\right\rangle \in R_{1}^{\mathcal{I}(0)}$, from which $e_{i} \in E_{i}^{\mathcal{I}(0)}$ and $e_{i}^{\prime}, e_{i}^{\prime \prime} \in E_{i}^{\dot{\mathcal{J}}(0)}$, for $1 \leq i \leq k$. Let $\mathrm{CARD}_{R}\left(R_{1}, U_{i}, E_{i}\right)=(\alpha, \beta)$. It is trivially satisfied for $t \neq 0$. Consider $t=0$ and let $e_{i}^{\prime} \in E_{i}^{\mathcal{J}(0)}$, so that $e_{i} \in E_{i}^{\mathcal{I}(0)}$. By the construction,

$$
\sharp\left\{\left\langle\tilde{e}_{1}, \tilde{e}_{2}, \ldots, \tilde{e}_{k}\right\rangle \in R_{1}^{\mathcal{J}(0)} \mid \tilde{e}_{i}=e_{i}^{\prime}\right\}=\sharp\left\{\left\langle\tilde{e}_{1}, \tilde{e}_{2}, \ldots, \tilde{e}_{k}\right\rangle \in R_{1}^{\mathcal{I}(0)} \mid \tilde{e}_{i}=e_{i}\right\} .
$$

As $\mathcal{I}$ satisfies the cardinality constraint, $\mathcal{J}$ satisfies it as well. Refinement constraints $\operatorname{REF}\left(R, U_{i}, E_{i}^{\prime}\right)=(\alpha, \beta)$ are considered similarly.

The construction for re-marking implicitly temporal attributes in $\Sigma$ as temporary ones is analogous. By repeating the above process sufficiently many times we obtain $\Sigma^{\prime}$ containing neither implicitly temporal relationships nor implicitly temporal attributes. 
Our first application of Lemma 1 is the following:

Theorem 1. Entity consistency for $E R_{\text {bool }}^{\mathrm{TS} \text { TRANS,EVO }}, E R_{\text {bool }}^{\mathrm{EVO}, \mathrm{TRANS}}, E R_{\text {bool }}^{\mathrm{TS} \text { TRANS }}$ and $E R_{\text {bool }}^{\mathrm{TRANS}}$ is PSPACE-complete.

Proof. The upper bound for $E R_{\text {bool }}^{\text {TS,TRANS,EVO }}$ follows from Lemma 1 and PSPACE-completeness of reasoning in $T_{F P X} D L-L i t e_{b o o l}^{\mathcal{N}}$ [6, Theorem 2]. The lower bound for $E R_{b o o l}^{\text {TRANS }}$ is an immediate consequence of the observation that entity consistency in this language (even without relationships and attributes) is capable of encoding satisfiability of propositional temporal formulas of the form $\theta \wedge \circledast \bigwedge_{i}\left(\varphi_{i} \rightarrow \bigcirc_{F} \psi_{i}\right)$, where $\theta$, the $\varphi_{i}$ and $\psi_{i}$ are conjunctions of literals. The latter problem is known to be PSPACE-hard [22].

Next, we observe that only the transition constraints TRANS require the next- and previous-time operators $\bigcirc_{F}$ and $\bigcirc_{P}$ in $\mathcal{T}_{\Sigma}$. Without these operators, satisfiability w.r.t. $T_{F P X} D L-L i t e_{b o o l}^{\mathcal{N}}$ TBoxes becomes NP-complete [6, Theorem 2], and so we obtain the following:

Theorem 2. Entity consistency for $E R_{\text {bool }}^{\mathrm{TS}, \mathrm{EVO}}, E R_{\text {bool }}^{\mathrm{EVO}}$ and $E R_{\text {bool }}^{\mathrm{TS}}$ is NP-complete.

Proof. Hardness follows from NP-completeness of entity consistency in $E R_{\text {bool }}$ [2].

We consider now temporal extensions of $E R_{\text {ref. }}$. Note that the Boolean $\sqcup$ is needed in $\mathcal{T}_{\Sigma}$ only to encode the covering, and that $\square$ in the translation of DEV can be easily eliminated, while the $⿴ 囗 \otimes)$ used to encode TS can be rewritten using $\square_{F}$ and $\square_{P}\left(\diamond_{F}, \diamond_{P}\right)$, e.g., a snapshot entity is translated as $E \sqsubseteq \square_{P} E$ and $E \sqsubseteq \square_{F} E$. This gives us an embedding of $E R_{\text {ref }}^{\text {TS TRANS,EVO }}$ into the fragment $T_{F P X} D L-L i t e_{c o r e}^{\mathcal{N}}$ of $T_{F P X} D L-L i t e_{b o o l}^{\mathcal{N}}$, reasoning in which is NP-complete [6, Theorem 3]. Concept inclusions of $T_{F P X} D L-L i t e_{\text {core }}^{\mathcal{N}}$ can only be of the form $D_{1} \sqsubseteq D_{2}$ and $D_{1} \sqcap D_{2} \sqsubseteq \perp$, where the $D_{i}$ are defined by the rule:

$$
D::=B\left|\diamond_{F} B\right| \diamond_{P} B\left|\square_{F} B\right| \square_{P} B\left|\bigcirc_{F} B\right| \bigcirc_{P} B .
$$

Theorem 3. Entity consistency for $E R_{r e f}^{\mathrm{TS}, \mathrm{TRANS}, \mathrm{EVO}}$ and $E R_{r e f}^{\mathrm{TRANS}, \mathrm{EVO}}$ is NP-complete.

Proof. The NP-hardness is proved by reduction of the NP-complete 3-colourability problem: given a graph $G=(V, E)$ and three colours $\{1,2,3\}$, decide whether there is an assignment of colours to the vertices in $V$ such that no two vertices sharing the same edge have the same colour. To reduce this problem to entity consistency in $E R_{\text {ref }}^{\mathrm{EVO}}$, we need entity symbols $O, X_{1}, X_{2}, X_{3}, Y$ and $A_{i}$, for $a_{i} \in V$. One possible way of encoding the graph $G$ by means of an $E R_{r e f}^{\mathrm{Evo}}$ schema $\Sigma_{G}$ is shown in Fig. 3.

Finally, consider $E R_{\text {ref }}$ extended with timestamping and transition constraints. In this case, the embedding into $T_{F P X} D L-L i t e_{c o r e}^{\mathcal{N}}$ uses only the next- $\bigcirc_{F}$ and previoustime $\bigcirc_{P}$ as well as 'at all moments' $\circledast$ and 'at some moment' $\otimes$ temporal operators, which makes reasoning tractable:

Theorem 4. Entity consistency for $E R_{\text {ref }}^{\text {TS,TRANS }}$ is in PTIME.

Proof. The proof can be found in the Appendix. 


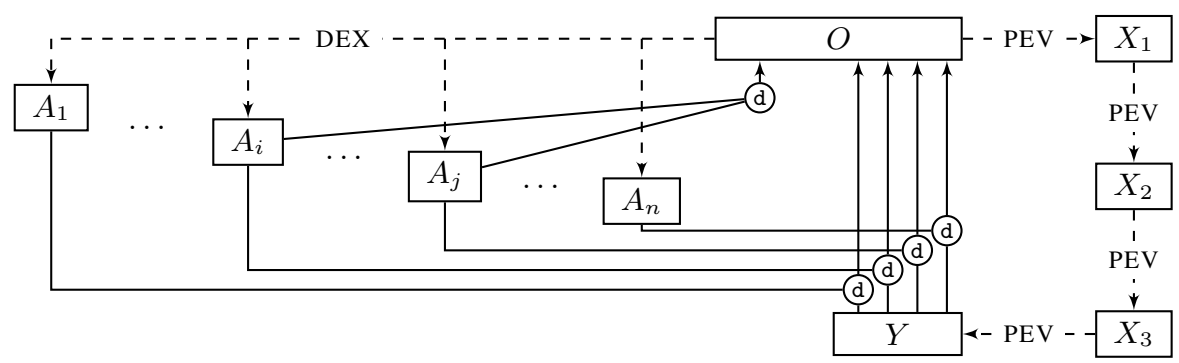

Fig. 3. 3-colourability in $E R_{r e f}^{\mathrm{EVO}}: A_{i}, A_{j}$ are disjoint sub-entities of $O$ for each $\left(a_{i}, a_{j}\right) \in E$.

Now, if we disallow the TRANS constraints (and so the use of $\bigcirc_{F}$ and $\bigcirc_{P}$ ), then reasoning becomes even simpler:

Theorem 5. Entity consistency for $E R_{\text {ref }}^{\mathrm{TS}}$ is NLOGSPACE-complete.

Proof. The upper bound follows from [6, Theorem 4], while the lower bound from the fact that already $E R_{r e f}$ is NLOGSPACE-complete [2].

\section{Undecidable Temporal Extensions of $E R_{\text {full }}$}

Theorem 6. Entity consistency for $E R_{\text {full }}^{\mathrm{TS}, \mathrm{TRANS}}$ and $E R_{\text {full }}^{\mathrm{EVO}, \mathrm{LFC}}$ is undecidable.

Proof. As shown in [2, Lemma 1], atemporal $E R_{\text {full }}$ is capable of expressing the qualified existential and universal restrictions $\exists R . C$ and $\forall R . C$ with their standard description logic semantics $(\exists R . C)^{\mathcal{I}}=\left\{x \in \Delta^{\mathcal{I}} \mid \exists y\left((x, y) \in R^{\mathcal{I}} \wedge y \in C^{\mathcal{I}}\right)\right\}$ and $(\forall R . C)^{\mathcal{I}}=\left\{x \in \Delta^{\mathcal{I}} \mid \forall y\left((x, y) \in R^{\mathcal{I}} \rightarrow y \in C^{\mathcal{I}}\right)\right\}$. These constructs together with the next-time operator on concepts and a single rigid role are enough to encode the undecidable tiling problem; see, e.g., [5]. It follows that $E R_{\text {full }}^{\mathrm{TS}, \mathrm{TRANS}}$ is undecidable.

As shown in [1, Theorem 5.2], $E R_{\text {full }}^{\mathrm{TS}, \mathrm{EVO}}$ is undecidable. A close inspection of the proof reveals that timestamping is required only to declare one snapshot entity, $O$, representing the whole domain, and one binary snapshot relationship, which is interpreted as a total functional time-invariant relation. The EVO constraints $O \mathrm{PEX} O$ and $O \mathrm{PEX}^{-} O$ and the IS A constraints $A$ ISA $O$, for each entity symbol $A$, make $O$ a snapshot entity representing the whole domain. Cardinality and lifespan cardinality constraints can make $R$ with $\operatorname{REL}(R)=\left(U_{1}: O, U_{2}: O\right)$ as required (see Fig. 4):

$$
\operatorname{CARD}_{R}\left(R, U_{1}, O\right)=(1, \infty) \quad \text { and } \quad \operatorname{L-CARD}\left(R, U_{1}, O\right)=(0,1) .
$$

It follows that $E R_{\text {full }}^{\mathrm{EVO}, \mathrm{LFC}}$ is undecidable.

\section{Lifespan Cardinality Constraints over $\boldsymbol{E} \boldsymbol{R}_{\text {bool }}$}

Let us consider now the case when lifespan cardinality constraints are required. Reasoning in $E R_{\text {full }}^{\mathrm{TS} \text { LFC }}$ is known to be 2EXPTIME-complete [8]. Here we show first that entity 


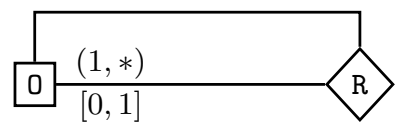

Fig. 4. Capturing functional total snapshot relationships using L-CARD.

consistency for $E R_{\text {bool }}^{\mathrm{TS} \text { LFC }}$ with binary relationships only is NP-complete. As in Section 3, the upper bound is proved by embedding the schema language into an appropriate temporal description logic, where traditionally only binary relations are used. So far, we used reification to encode relationships of arbitrary arity; see (R) in Section 3. However, this encoding does not preserve the meaning of timestamped relationships in the presence of lifespan cardinality constraints. Indeed, Lemma 2 does not hold anymore and we cannot disregard the difference between temporary and implicitly temporal relationships (to see this, note that if we constraint the relationship $R$ in the schema in Fig. 4 to be temporary then the schema becomes inconsistent). On the other hand, the reification employed in [8] for capturing $n$-ary temporary relationships for the $E R_{\text {full }}^{\mathrm{TS}, \mathrm{LFC}}$ language was based on a temporal extension of $\mathcal{A L C}$ and it does not work for the much simpler temporal DL-Lite logics. That is why we restrict the language to binary relationships.

The variant of NP-complete temporal $D L$-Lite we need is called $T_{U}^{\mathcal{R}} D L-L i t e_{\text {bool }}^{\mathcal{N}}$ [6]. It uses the 'universal modalities' $*$ and $*$ on both concepts and roles. The semantics of temporalised roles $\circledast R$ and $\otimes R$ (required to encode LFC) is defined as follows:

$$
(\circledast R)^{\mathcal{I}(n)}=\bigcap_{k \in \mathbb{Z}} R^{\mathcal{I}(k)} \quad \text { and } \quad(\otimes R)^{\mathcal{I}(n)}=\bigcup_{k \in \mathbb{Z}} R^{\mathcal{I}(k)} .
$$

Given an $E R_{\text {bool }}^{\mathrm{TS} \text { LFC }}$ schema $\Sigma$ (with binary relations only), we construct a $T_{U}^{\mathcal{R}} D L-L i t e_{b o o l}^{\mathcal{N}}$ TBox $\mathcal{T}_{\Sigma}$ in a way similar to the translation in Section 3. As before, all entity and domain symbols in $\Sigma$ are regarded as concept names in $\mathcal{T}_{\Sigma}$. However, the relationship and attribute symbols in $\Sigma$ will now be regarded as role names in $\mathcal{T}_{\Sigma}$; the role symbols in $\Sigma$ will have no counterparts in $\mathcal{T}_{\Sigma}$. We define $\mathcal{T}_{\Sigma}$ as $\mathcal{T}_{\Sigma}^{0} \cup \mathcal{T}_{\Sigma}^{1}$, where $\mathcal{T}_{\Sigma}^{0}$ encodes the atemporal constructs and $\mathcal{T}_{\Sigma}^{1}$ the temporal ones. $\mathcal{T}_{\Sigma}^{0}$ is defined as in Section 3 with the exception of (R) which is replaced with the following:

$\left(\mathbf{R}^{\prime}\right)$ for $\operatorname{REL}(R)=\left\langle U_{1}: E_{1}, U_{2}: E_{2}\right\rangle$,

- $\exists R \sqsubseteq E_{1}$ and $\exists R^{-} \sqsubseteq E_{2}$,

- $E_{1} \sqsubseteq \geq \alpha R$ and $E_{1} \sqsubseteq \leq \beta R$ if $\operatorname{CARD}_{R}\left(R, U_{1}, E_{1}\right)=(\alpha, \beta)$,

- $E_{2} \sqsubseteq \geq \alpha R^{-}$and $E_{2} \sqsubseteq \leq \beta R^{-}$if $\operatorname{CARD}_{R}\left(R, U_{2}, E_{2}\right)=(\alpha, \beta)$,

- $E_{1}^{\prime} \sqsubseteq \geq \alpha R$ and $E_{1}^{\prime} \sqsubseteq \leq \beta R$ if $\operatorname{REF}\left(R, U_{1}, E_{1}^{\prime}\right)=(\alpha, \beta)$,

- $E_{2}^{\prime} \sqsubseteq \geq \alpha R^{-}$and $E_{2}^{\prime} \sqsubseteq \leq \beta R^{-}$if $\operatorname{REF}\left(R, U_{2}, E_{2}^{\prime}\right)=(\alpha, \beta)$.

The temporal part $\mathcal{T}_{\Sigma}^{1}$ contains the concept inclusions:

(TS) $\top \sqsubseteq \neg \exists$ ⿶ $R$ for temporary $R \in \mathcal{R}^{\mathrm{T}}$; and the role $R$ is rigid for snapshot $R \in \mathcal{R}^{\mathrm{S}}$, $\top \sqsubseteq \neg \exists$ $凵 A$ for temporary $A \in \mathcal{A}^{\top}$; and the role $A$ is rigid for snapshot $A \in \mathcal{A}^{\mathrm{S}}$;

(LFC) for $\operatorname{REL}(R)=\left\langle U_{1}: E_{1}, U_{2}: E_{2}\right\rangle$, 
- $E_{1} \sqsubseteq \geq \alpha \otimes R$ and $E_{1} \sqsubseteq \leq \beta \otimes R$ if $\operatorname{L-CARD}\left(R, U_{1}, E_{1}\right)=(\alpha, \beta)$,

- $E_{2} \sqsubseteq \geq \alpha \otimes R^{-}$and $E_{2} \sqsubseteq \leq \beta \otimes R^{-}$if $\operatorname{L-CARD}\left(R, U_{2}, E_{2}\right)=(\alpha, \beta)$,

- $E_{1}^{\prime} \sqsubseteq \geq \alpha \otimes R$ and $E_{1}^{\prime} \sqsubseteq \leq \beta \otimes R$ if $\operatorname{L-REF}\left(R, U_{1}, E_{1}^{\prime}\right)=(\alpha, \beta)$,

- $E_{2}^{\prime} \sqsubseteq \geq \alpha \otimes R^{-}$and $E_{2}^{\prime} \sqsubseteq \leq \beta \otimes R^{-}$if $\operatorname{L-REF}\left(R, U_{2}, E_{2}^{\prime}\right)=(\alpha, \beta)$.

( $E \sqsubseteq \geq \alpha S$ with $\alpha=0$ and $E \sqsubseteq \leq \beta S$ with $\beta=\infty$ are not included in $\mathcal{T}_{\Sigma}$.)

Lemma 3. An entity $E$ is consistent w.r.t. an $E R_{\text {bool }}^{\mathrm{TS}, \mathrm{LFC}}$ schema $\Sigma$ with binary relationships if and only if $E$ is satisfiable w.r.t. the $T_{U}^{\mathcal{R}} D L-L i t e_{\text {bool }}^{\mathcal{N}} T B o x \mathcal{T}_{\Sigma}$.

Proof. The proof is straightforward and left to the reader.

Using Lemma 3 and NP-completeness of $T_{U}^{\mathcal{R}} D L-L i t e_{\text {bool }}^{\mathcal{N}}$ [6, Theorem 6], we obtain:

Theorem 7. Entity consistency for $E R_{\text {bool }}^{\mathrm{TS}, \mathrm{LFC}}$ with binary relationships is NP-complete.

Proof. The lower bound follows immediately from NP-completeness of $E R_{b o o l}$ [2].

Unfortunately, if we extend $E R_{b o o l}^{\mathrm{LFC}}$ (with binary relationships) by means of the transition constraints, the resulting language becomes undecidable.

Theorem 8. Entity consistency for $E R_{\text {bool }}^{\mathrm{TRANS}, \mathrm{LFC}}$ is undecidable.

Proof. We recall from [6, Theorem 5] that if we slightly modify $T_{U}^{\mathcal{R}} D L-L i t e_{\text {bool }}^{\mathcal{N}}$ by replacing the operators $\otimes$ and $\otimes$ on concepts with $\bigcirc_{F}$, and keeping the same temporalised roles, then the resulting logic $T_{X}^{\mathcal{R}} D L-L i t e_{\text {bool }}^{\mathcal{N}}$ is undecidable. A close inspection of the proof reveals that to prove undecidability we need, apart from a number of concept names, a single role name $P$, which occurs in concepts $\exists P$ and $\exists P^{-}$and two functionality axioms $\geq 2 \otimes P \sqsubseteq \perp$ and $\geq 2 \otimes P^{-} \sqsubseteq \perp$. We show that such a TBox $\mathcal{T}$ can be transformed into an equisatisfiable TBox $\mathcal{T}^{\prime}$, which contains the two functionality axioms and only CIs of the form $\top \sqsubseteq A$ and $A \sqsubseteq C$, where $C$ is a concept of the form

$$
C::=A|\neg A| A_{1} \sqcup A_{2}|\exists P| \exists P^{-} \mid \bigcirc_{F} A,
$$

and $A, A_{1}$ and $A_{2}$ are concept names. Indeed, every CI, $C_{1} \sqsubseteq C_{2}$, can be rewritten as $\top \sqsubseteq A$ and $A \sqsubseteq \neg C_{1} \sqcup C_{2}$, where $A$ is a fresh concept name. We transform the right-hand side of $A \sqsubseteq \neg C_{1} \sqcup C_{2}$ into negation normal form and then recursively apply the following rules:

1. $A \sqsubseteq C_{1} \sqcap C_{2}$ is replaced by $A \sqsubseteq C_{1}$ and $A \sqsubseteq C_{2}$,

2. $A \sqsubseteq C_{1} \sqcup C_{2}$ is replaced by $A \sqsubseteq A_{1} \sqcup A_{2}, A_{1} \sqsubseteq C_{1}, A_{2} \sqsubseteq C_{2}$ (with fresh $A_{i}$ ),

3. $A \sqsubseteq \bigcirc_{F} C$ is replaced by $A \sqsubseteq \bigcirc_{F} A_{1}$ and $A_{1} \sqsubseteq C$ (with fresh $A_{1}$ ).

The resulting TBox is the required $\mathcal{T}^{\prime}$. Now we show that concept satisfiability w.r.t. $\mathcal{T}^{\prime}$ can be reduced to entity consistency w.r.t. the $E R_{\text {bool }}^{\text {TRANSLFC }}$ schema $\Sigma_{\mathcal{T}^{\prime}}$. The reduction is similar to the one used in [10]. Concept names in $\mathcal{T}^{\prime}$ are regarded as entity symbols in $\Sigma_{\mathcal{T}^{\prime}} ; \top$ is represented by a fresh entity symbol $O$ with $A$ IS A $O$, for each entity symbol $A$; time-invariance of $O$ is expressed by $O$ TEX $O$ and $O$ TEX $^{-} O$. The role name $P$ 
in $\mathcal{T}^{\prime}$ is regarded as a relationship symbol $P$ with $\operatorname{REL}(P)=\left\langle U_{1}: O, U_{2}: O\right\rangle$. The functionality of $\otimes P$ and $\otimes P^{-}$is enforced by the constraints, cf. (3):

$$
\operatorname{L-CARD}\left(P, U_{1}, O\right)=(0,1) \quad \text { and } \quad \operatorname{L-CARD}\left(P, U_{2}, O\right)=(0,1) \text {. }
$$

Concept inclusions of the form $\top \sqsubseteq A$ and $A \sqsubseteq A_{1}$ are encoded as $O$ ISA $A$ and $A$ ISA $A_{1}$. For encoding of CIs of the form $A \sqsubseteq \neg A_{1}$ and $A \sqsubseteq A_{1} \sqcup A_{2}$ we refer the reader to [10, Theorem 5.6]. Additionally, we take two fresh entities $E_{\exists P}$ and $E_{\exists P^{-}}$ and extend the schema with the following constraints:

- $A$ ISA $E_{\exists P}$ and $\operatorname{REF}\left(P, U_{1}, E_{\exists P}\right)=(1, \infty)$, for $A \sqsubseteq \exists P$ in $\mathcal{T}^{\prime}$,

- $A$ ISA $E_{\exists P^{-}}$and $\operatorname{REF}\left(P, U_{2}, E_{\exists P^{-}}\right)=(1, \infty)$, for $A \sqsubseteq \exists P^{-}$in $\mathcal{T}^{\prime}$,

- $A$ TEX $A_{1}$, for $A \sqsubseteq \bigcirc_{F} A$ in $\mathcal{T}^{\prime}$.

Using a technique similar to [10, Theorem 5.6], one can show that, for each concept name $A, A$ is satisfiable w.r.t. $\mathcal{T}^{\prime}$ if and only if $A$ is consistent w.r.t. $\Sigma_{\mathcal{T}}$.

\section{Conclusion}

We have investigated the computational complexity of checking quality properties of temporal conceptual models such as entity, relationship and schema satisfiability or entity/relationship subsumption. Although 'negative' (undecidability) results were known for temporal extensions of the full (atemporal) UML/EER, we have found fragments with a much better computational behaviour by considering the language $E R_{\text {bool }}$ where IS A between relationships is disallowed, and its sub-language $E R_{\text {ref }}$ where covering constraints are not available. These languages have been extended with timestamping, evolution and transition constraints, and lifespan cardinalities. We have obtained a nearly comprehensive classification of the computational complexity of reasoning over the resulting temporal conceptual models, which is summarised in Table 2. Three cases involving lifespan cardinalities still remain open. In particular, we do not know whether $E R_{\text {ref }}$ with lifespan cardinalities and transition (or evolution) constraints is decidable. Another interesting question we are working on now is whether standard temporal provers, quantified Boolean or SAT solvers can be used for efficient practical reasoning over temporal conceptual models.

\section{References}

1. A. Artale. Reasoning on temporal class diagrams: Undecidability results. Annals on Mathematics and Artificial Intelligence (AMAI), 46:265-288, 2006. Springer.

2. A. Artale, D. Calvanese, R. Kontchakov, V. Ryzhikov, and M. Zakharyaschev. Reasoning over extended ER models. In Proc. of the $26^{\text {th }}$ Int. Conf. on Conceptual Modeling (ER'07). Lecture Notes in CS, Springer, 2007.

3. A. Artale, D. Calvanese, R. Kontchakov, and M. Zakharyaschev. The DL-Lite family and relations. Journal of Artificial Intelligence Research, 36:1-69, 2009.

4. A. Artale, E. Franconi, and F. Mandreoli. Description logics for modelling dynamic information. In Jan Chomicki, Ron van der Meyden, and Gunter Saake, editors, Logics for Emerging Applications of Databases. Lecture Notes in Computer Science, Springer-Verlag, 2003. 
5. A. Artale, E. Franconi, F. Wolter, and M. Zakharyaschev. A temporal description logic for reasoning about conceptual schemas and queries. In Proc. of the $8^{\text {th }}$ Joint European Conference on Logics in Artificial Intelligence (JELIA-02), LNAI, 2002.

6. A. Artale, R. Kontchakov, V. Ryzhikov, and M. Zakharyaschev. Past and future of DL-Lite. In Proc. of the $24^{\text {th }}$ AAAI Conf. on Artificial Intelligence (AAAI-10), 2010.

7. A. Artale, C. Parent, and S. Spaccapietra. Evolving objects in temporal information systems. Annals of Mathematics and Artificial Intelligence, 50(1-2):5-38, 2007.

8. A. Artale and D. Toman. Decidable reasoning over timestamped conceptual models. In Proc. of the 21st Int. Workshop on Description Logics (DL-08), Dresden, Germany, May 2008.

9. F. Baader, D. Calvanese, D. McGuinness, D. Nardi, and P. F. Patel-Schneider, editors. Description Logic Handbook: Theory, Implementation and Applications. Cambridge University Press, 2002.

10. D. Berardi, D. Calvanese, and G. De Giacomo. Reasoning on UML class diagrams. Artificial Intelligence, 168(1-2):70-118, 2005.

11. D. Calvanese, M. Lenzerini, and D. Nardi. Unifying class-based representation formalisms. J. of Artificial Intelligence Research, 11:199-240, 1999.

12. C. Combi, S. Degani, and C. S. Jensen. Capturing temporal constraints in temporal ER models. In Proc. of the $27^{\text {th }}$ Int. Conf. on Conceptual Modeling (ER'08). LNCS, 2008.

13. O. Etzion, A. Gal, and A. Segev. Extended update functionality in temporal databases. In Temporal Databases - Research and Practice. LNCS, 1998.

14. H. Gregersen and J.S. Jensen. Conceptual modeling of time-varying information. Technical Report TimeCenter TR-35, Aalborg University, Denmark, 1998.

15. H. Gregersen and J.S. Jensen. Temporal Entity-Relationship models - a survey. IEEE Transactions on Knowledge and Data Engineering, 11(3):464-497, 1999.

16. R. Gupta and G. Hall. An abstraction mechanism for modeling generation. In Proc. of ICDE'92, pages 650-658, 1992.

17. G. Hall and R. Gupta. Modeling transition. In Proc. of ICDE'91, pages 540-549, 1991.

18. C. S. Jensen, J. Clifford, S. K. Gadia, P. Hayes, and S. Jajodia et al. The Consensus Glossary of Temporal Database Concepts. In Temporal Databases - Research and Practice. SpringerVerlag, 1998.

19. C. S. Jensen and R. T. Snodgrass. Temporal data management. IEEE Transactions on Knowledge and Data Engineering, 111(1):36-44, 1999.

20. C. S. Jensen, M. Soo, and R. T. Snodgrass. Unifying temporal data models via a conceptual model. Information Systems, 9(7):513-547, 1994.

21. C. Lutz, F. Wolter, and M. Zakharyaschev. Temporal description logics: A survey. In Proc. of TIME 2008, 2008.

22. A. Prasad Sistla and Edmund M. Clarke. The complexity of propositional linear temporal logics. In Proc. of the $14^{\text {th }}$ Annual ACM Symposium on Theory of Computing. ACM, 1982.

23. S. Spaccapietra, C. Parent, and E. Zimanyi. Conceptual Modeling for Traditional and SpatioTemporal Applications-The MADS Approach. Springer, 2006.

24. B. Tauzovich. Towards temporal extensions to the entity-relationship model. In Proc. of the Int. Conf. on Conceptual Modeling (ER'91). Springer-Verlag, 1991.

25. C. Theodoulidis, P. Loucopoulos, and B. Wangler. A conceptual modelling formalism for temporal database applications. Information Systems, 16(3):401-416, 1991. 


\section{A Proof of Theorem 4}

Theorem 4. Entity consistency for $E R_{\text {ref }}^{\mathrm{TS} \text { TRANS }}$ is decidable in PTIME.

Proof. By Lemma 1, the entity consistency problem can be reduced to concept satisfiability with respect to $T_{F P X} D L-L i t e_{b o o l}^{\mathcal{N}}$ TBoxes. The latter, in turn, is reducible to the satisfiability problem in propositional temporal logic; cf. [TR, Theorem 1]. Here we give a slightly modified version of that reduction (the main difference from [TR] is that now the reduction is deterministic).

First, we reduce satisfiability of a $T_{F P X} D L-L i e_{b o o l}^{\mathcal{N}} \operatorname{KB} \mathcal{K}=(\mathcal{T}, \mathcal{A})$ to satisfiability in the one-variable first-order temporal logic in a way similar to [FR]. For each basic concept $B(\neq \perp)$, we take a fresh unary predicate $B^{*}(x)$ and encode $\mathcal{T}$ as

$$
\mathcal{T}^{\dagger}=\bigwedge_{C_{1} \sqsubseteq C_{2} \in \mathcal{T}} \circledast \forall x\left(C_{1}^{*}(x) \rightarrow C_{2}^{*}(x)\right),
$$

where the $C_{i}^{*}$ are the results of replacing each $B$ with $B^{*}(x)$ ( $\sqcap$ with $\wedge$, etc.). We assume that $\mathcal{T}$ contains CIs of the form $\geq q R \sqsubseteq \geq q^{\prime} R$, for $\geq q R, \geq q^{\prime} R$ in $\mathcal{T}$ such that $q>q^{\prime}$ and there is no $q^{\prime \prime}$ with $q>q^{\prime \prime}>q^{\prime}$ and $\geq q^{\prime \prime} R$ in $\mathcal{T}$. We also assume that $\mathcal{T}$ contains $\geq q R \equiv \otimes \geq q R$ if $\geq q R$ occurs in $\mathcal{T}$, for a rigid role $R$ (i.e., for $G_{i}$ or $G_{i}^{-}$). To take account of the fact that roles are binary relations, we add to $\mathcal{T}^{\dagger}$ the following formula, for each role name $S$,

$$
\varepsilon_{S}=\circledast\left(\exists x(\exists S)^{*}(x) \leftrightarrow \exists x\left(\exists S^{-}\right)^{*}(x)\right)
$$

(which says that at each moment of time the domain of $S$ is nonempty iff its range is nonempty). The ABox $\mathcal{A}$ is encoded by a conjunction $\mathcal{A}^{\dagger}$ of ground atoms of the form $\bigcirc^{m} B^{*}(a)$ and $\bigcirc^{n}(\geq q R)^{*}(a)$ in the same way as in [FR]. Thus, $\mathcal{K}$ is satisfiable iff the formula

$$
\mathcal{K}^{\dagger}=\mathcal{T}^{\dagger} \wedge \bigwedge_{S} \varepsilon_{S} \wedge \mathcal{A}^{\dagger}
$$

is satisfiable.

The second step of our reduction is based on the observation that if $\mathcal{K}^{\dagger}$ is satisfiable then it can be satisfied in an interpratation such that

$\left(\mathbf{R}_{0}\right)$ if $(\exists S)^{*}(x)$ is true at some moment (on some domain element) then it is true at 0 (perhaps on a different domain element).

Indeed, if $\mathcal{K}^{\dagger}$ is satisfied in $\mathcal{I}$ then it is satisfied in the disjoint union $\mathcal{I}^{*}$ of all $\mathcal{I}^{n}$, $n \in \mathbb{Z}$, obtained from $\mathcal{I}$ by shifting its time line $n$ moments forward. Therefore, if $(\exists S)^{*}(x)$ is true at some moment of time on some element, then there is an element such that $(\exists S)^{*}(x)$ is true on it at 0 . It follows then from $\left(\mathbf{R}_{0}\right)$ that $\mathcal{K}^{\dagger}$ is satisfiable iff the formula

$$
\begin{aligned}
\mathcal{K}^{\ddagger}=\mathcal{T}^{\dagger} \wedge & \bigwedge_{S}\left(\left(p_{S} \rightarrow(\exists S)^{*}\left(d_{S}\right)\right) \wedge\left(p_{S} \rightarrow\left(\exists S^{-}\right)^{*}\left(d_{S^{-}}\right)\right)\right) \wedge \\
& \bigwedge_{S} \circledast \forall x\left(\left((\exists S)^{*}(x) \rightarrow \circledast p_{S}\right) \wedge\left(\left(\exists S^{-}\right)^{*}(x) \rightarrow \boxplus p_{S}\right)\right) \wedge \mathcal{A}^{\dagger}
\end{aligned}
$$


is satisfiable, where the $p_{S}$ are fresh propositional variables and the $d_{S}$ and $d_{S^{-}}$are fresh constants (informally, the role $S$, with $p_{S}$ being false at 0 , are always empty, whereas other roles are nonempty at 0 , and so are always nonempty). Finally, as $\mathcal{K}^{\ddagger}$ contains no existential quantifiers, it can be regarded as a propositional temporal formula because all the universal quantifiers can be instantiated by all the constants in the formula, which results only in a polynomial blow-up of $\mathcal{K}^{\ddagger}$.

It turns out that entity consistency in $E R_{r e f}^{\text {TS,TRANS }}$ can in fact be reduced to satisfiability of propositional temporal formulas of the form

$$
\bigwedge \Psi \wedge \bigwedge \circledast \Phi \wedge \bigwedge \Theta
$$

where $\Psi$ and $\Phi$ are sets of unary and binary Horn clauses of the form

$$
p, \quad p \rightarrow p^{\prime}, \quad \neg p \vee \neg p^{\prime}, \quad p \rightarrow \bigcirc_{F} p^{\prime} \quad \text { or } \quad \bigcirc_{F} p \rightarrow p^{\prime}
$$

(where $p$ and $p^{\prime}$ are propositional variables), $\circledast \Phi$ is the result of prefixing each clause in $\Phi$ with 赵, and $\Theta$ is a set of formulas of the form $\otimes p \rightarrow \circledast p^{\prime}$. For such formulas we have the following satisfiability criterion:

Lemma 4. Let $\varphi$ be a propositional temporal formula of the form $\wedge \Psi \wedge \wedge \circledast \Phi \wedge \wedge \Theta$ and let

$$
\Theta_{0}=\left\{p^{\prime} \mid \circledast p \rightarrow \circledast p^{\prime} \in \Theta \text { and }(\bigwedge \Psi \wedge \bigwedge \circledast \Phi) \models \circledast p\right\} .
$$

Then $\varphi$ is satisfiable if and only if $\bigwedge \Psi \wedge \bigwedge \circledast \Phi \wedge \bigwedge \circledast \Theta_{0}$ is satisfiable.

Proof. $\left(\Rightarrow\right.$ ) Let $\mathfrak{M}, 0 \models \varphi$. Then $\mathfrak{M}, 0 \models \wedge \Psi \wedge \bigwedge \circledast \Phi$ and, by the definition of $\Theta_{0}$, $\mathfrak{M}=\circledast p$, for each $\otimes p \rightarrow \circledast p^{\prime} \in \Theta$ with $p^{\prime} \in \Theta_{0}$. Therefore, $\mathfrak{M}=\Lambda \circledast \Theta_{0}$.

$(\Leftarrow)$ For each $\otimes p$ with $\boxplus p \rightarrow \boxplus p^{\prime} \in \Theta$ and $p^{\prime} \notin \Theta_{0}$, take a model $\mathfrak{M}_{p}$ such that $\mathfrak{M}_{p}, 0 \models \bigwedge \Psi \wedge \bigwedge \circledast \Phi \wedge \neg \circledast p$. Also, take a model $\mathfrak{M}^{\prime}$ satisfying $\Lambda \Psi \wedge \bigwedge \circledast \Phi \wedge \bigwedge \circledast \Theta_{0}$ at 0 . Construct now a model $\mathfrak{M}$ by taking the intersection of $\mathfrak{M}^{\prime}$ and the $\mathfrak{M}_{p}$ :

$$
\mathfrak{M}, m \models q \quad \text { iff } \quad \mathfrak{M}^{\prime}, m=q \text { and } \mathfrak{M}_{p}, m=q \text {, for each } \circledast p,
$$

for all moments $m \in \mathbb{Z}$ and propositional variables $q$. Since all clauses in $\Phi$ and $\Psi$ are Horn, $\mathfrak{M}, 0 \models \bigwedge \Psi \wedge \bigwedge \circledast \Phi$. It also follows from the construction that $\mathfrak{M}=\circledast p \rightarrow \circledast p^{\prime}$, for all $\circledast p \rightarrow \circledast p^{\prime} \in \Theta$, and so $\mathfrak{M}, 0 \models \varphi$.

Similarly to the proof of [TR, Theorem 3], define $\operatorname{cons}_{\Phi}^{m}(\Psi)$ by taking

$$
\begin{aligned}
& \operatorname{cons}_{\Phi}^{0}(\Psi)=\{L \mid \Phi \cup \Psi \models L\}, \\
& \operatorname{cons}_{\Phi}^{m}(\Psi)=\left\{L \mid \Phi=L^{\prime} \rightarrow \bigcirc_{F} L, L^{\prime} \in \operatorname{cons}_{\Phi}^{m-1}(\Psi)\right\} \cup\{L \mid \Phi \models L\} \text { if } m \geq 1, \\
& \operatorname{cons}_{\Phi}^{m}(\Psi)=\left\{L \mid \Phi=L^{\prime} \rightarrow \bigcirc_{P} L, L^{\prime} \in \operatorname{cons}_{\Phi}^{m+1}(\Psi)\right\} \cup\{L \mid \Phi \models L\} \text { if } m \leq-1,
\end{aligned}
$$

where $L$ and $L^{\prime}$ are literals (variables or their negations). As in the proof of [TR, Theorem 3], since both $\Phi$ and $\Psi$ are essentially 2CNFs, we have $p \in \operatorname{cons}_{\Phi}^{m}(\Psi)$ if and only if $\mathfrak{M}, m \models p$, for every $\mathfrak{M}$ with $\mathfrak{M}, 0 \models \wedge \Psi \wedge \bigwedge \circledast \Phi$. Therefore, $\varphi$ is satisfiable just in case 
- for each propositional variable $q$, there is no $m \in \mathbb{Z}$ with $q, \neg q \in \operatorname{cons}_{\Phi \cup \Theta_{0}}^{m}(\Psi)$,

where $\Theta_{0}$ is constructed by taking the set of all $p^{\prime}$ such that $\circledast p \rightarrow \circledast p^{\prime} \in \Theta$ and there is no $m \in \mathbb{Z}$ with $\neg p \in \operatorname{cons}_{\Phi}^{m}(\Psi)$.

It remains to note that the set $\Theta_{0}$ can be constructed and the condition above can be verified in deterministic polynomial time by solving Diophantine equations for the arithmetic progressions constructed using Unary Finite Automata in the same way as in the proof of [TR, Theorem 3].

\section{References}

[TR] A. Artale, R. Kontchakov, V. Ryzhikov, and M. Zakharyaschev. Temporal conceptual modelling with DL-Lite. Technical Report BBKCS-10-02, Department of Computer Science and Information Systems, Birkbeck, University of London, March 2010.

[FR] A. Artale, R. Kontchakov, V. Ryzhikov, and M. Zakharyaschev. DL-Lite with temporalised concepts, rigid axioms and roles. In Proc. of FroCoS, 2009. 